\title{
Alternative Medical Procedure
}

National Cancer Institute

\section{Source}

National Cancer Institute. Alternative Medical Procedure. NCI Thesaurus. Code C64386.

Treatment used instead of, or supplementary to, conventional Western-based practices.

Alternative medicine includes treatments and practices such as acupuncture, holistic

medicine, homeopathy, ayurveda, Chinese medicine, massage therapy, herbal therapy,

and biofeedback. 